\title{
Review of: "The MRI-based 3D morphologic changes of knee meniscus under knee weight-bearing and early flexion conditions"
}

\author{
Gregorio Marchiori ${ }^{1}$ \\ 1 Istituto Ortopedico Rizzoli
}

Potential competing interests: The author(s) declared that no potential competing interests exist.

The authors of the paper declare that "this is the first study to investigate the three-dimensional morphologic change of human meniscus under loading and knee flexion condition using in vivo MRIcompatible loading device". It aims to be a reference for other studies that want to investigate meniscal function in a pathological context, but it could be useful also for evaluating the in vivo performances of meniscal regenerative/treatment solutions.

The intention is clear, but from my point of view not fully supported due to some weak aspects:

- Several grammar errors or vague expressions, starting from the Abstract, make it difficult for the reader to easily understand some passages, both for methods and results.

- Even if the Introduction says that "few studies used this approach to investigate the meniscus", the Discussion lacks a paragraph that list the advantages of this work against each specific cited reference. In fact, the approach seems to introduce some novelty respect to Stehling et al. (2012) and Patel et al. (2016), but what about Vedi et al. (1999)? If three-dimensionality, for instance, it is not specifically highlighted.

- Output parameters are not fully satisfactory:

1. Meniscus deformation seems to be an important parameter to be assessed: it is cited in the Abstract ("3D deformation and displacement of the whole meniscus between in vivo mechanical loading and unloading conditions are still unclear") and in the Introduction ("3D deformation and displacement of the whole meniscus ... are not clear"). In its quantitative form, that is strain, it represents a fundamental reference for meniscal tissue biomechanics, but the proposed methodological framework does not try to obtain it. It is substituted by a generic morphological "difference", or "change", or "deviation", not always clear in its definition (e.g., it is stated as negative also when the surface is moving close to the reference model) and calculated before displacements. Why not to calculate the displacements first, then - in order to compensate them - align the baseline and test menisci on their 
circle centre and finally calculate the morphological changes, thus closer to real deformation? Actually, it could be a better answer to the question advanced by the same authors when they say "it was not clear if those changes were made either from displacements or from deformations". Indeed, deformation/strain is used by various related studies (Freutel et al. (2014), Fowlie et al. (2011), Kessler et al. (2015))

2. A sketch for extrusion calculation, as Figure 2 for displacement, could have been useful for the reader.

- The structure of the comparisons is not clear: which and how are presented. For example, in the Statistical analyses paragraph the "positive/negative deviation" is combined with subregions and unloaded/loaded conditions, while we did not know how deviation maps were condensed in single values. The Results paragraph is confusing in several points. It is still not clear how mapped deviations are averaged to perform the comparisons (actually the terms "mean positive difference" and "mean negative difference" appear in the Appendix, but they should be described also in the main text); different subparagraphs for "general changes", "displacements" and "extrusion" would have been useful. The comparisons remain not fully clear in some points of the Discussion and in the Appendix.

- All recruited subjects were male: it should have been commented by the authors in the limitations, standing the gender differences in terms of anatomy and gait (e.g. see https://doi.org/10.1016/j.gaitpost.2020.05.017).

I am not an expert of statistics, but I appreciated the parametric-nonparametric specification and the power analysis in the Statistical paragraph: many articles neglect these aspects. 